\title{
The Magnitude of Unsafe Child Feces Disposal Practices and Its Association With Reported Diarrhea in Low-income and Middle-income Countries: A Systematic Review and Meta-analysis Protocol
}

Negasa Eshete Soboksa ( $\square$ yeroosaa@gmail.com )

Addis Ababa University https://orcid.org/0000-0003-3451-175X

\section{Bekam Kebede Olkeba}

Hawassa University College of Medicine and Health Sciences

\section{Dinkinesh Begna Gudeta}

Arsi University College of Health Sciences

\section{Protocol}

Keywords: magnitude, feces disposal, child, diarrhea

Posted Date: July 30th, 2020

DOI: https://doi.org/10.21203/rs.3.rs-50165/v1

License: (c) (1) This work is licensed under a Creative Commons Attribution 4.0 International License. Read Full License 


\section{Abstract}

Introduction:The unsafe disposal of children's feces may be an important contaminant in household environments, posing a high risk of exposure to infants. Several studies done on the magnitude of unsafe disposal of child feces and its association with reported childhood diarrheahave variedoutcomesand no tries have been made to systematically review this. Therefore, itis necessitating a systematic review to provide an exhaustive summary of current evidence. Thus, the objective ofthis study will be to pool out the available evidence on the magnitude of unsafe child feces disposalpractices and its association with reported childhood diarrhea in low-income and middle-income countries.

Methods: PubMed, Science Direct, Cochrane Library database, and Ovid Medline will be searched to identify relevant literature for this review. Moreover, Google search engine, Google Scholar, and references of other studieswill be searched from January 2000 to December 2020. The primary outcome of interest will bethe magnitude of unsafe disposal of child feces and the secondary outcome will be its association with reported diarrhea. Observationalstudies (cross-sectional studies, case-control studies, and cohort studies) written in English will be included in this review. The selected studies will be critically appraised by two independent reviewers using an appropriate tool. The pooled magnitude of unsafe disposal of child feces and its association with reported childhood diarrhea will be analyzed using Stata version 16. Heterogeneity will be assessed using the chi-square test (Q-test) statistics and inverse variance index $\left(I^{2}\right)$. Forest plots will be used to present the combined estimate with 95\% Cl.A funnel plot and Egger's test of small study bias will be used to assess publication bias.

Discussion:This systematic review will identify the evidence available on themagnitude of unsafe child fecesdisposal practicesand its associationwith reported diarrhea. The findings from this study will bemade publicly available in a repository and published in a peer-reviewed journal. The findings from this study will also provide directions for future research and public health professionals with an understanding of the importance of safe child feces disposal practices to preventingchildhood diarrhea in the community.

\section{Systematic review registrationnumber: PROSPERO CRD42020189034}

\section{Introduction}

Lack of sanitation facilities and poor hygiene practices cause millions of the world's poorest people to die from preventable diseases each year [1]. Many countries are challenged in providing adequate sanitation for their entire population and leaving people at risk [2]. Inadequate sanitation is estimated to cause 432,000 diarrhea deaths annually and is a major factor in several neglected tropical diseases, including intestinal worms, schistosomiasis, and trachoma and it also contributes to malnutrition [3]. The study revealed about 2 billion people living in rural areas are adversely affected by the lack of adequate water supply and by open defecation [4]. A study done by Prüss-Üstünet al. showed that $5.48 \%$ of all deaths 
and $7.67 \%$ of the disability-adjusted life years (DALYs) lost in low-income and middle-income countries are due to poor WASH-related diarrheal diseases and parasite infestations [5].

About 2.0 billion people still do not have basic sanitation facilities such as toilets or latrines. Of these, 673 million still defecate in the open, for example, in street gutters, behind bushes, or into open bodies of water [3]. In Ethiopia, access to improved sanitation facilities is also very limited in rural areas where the majority of households defecate in the bush or open fields [6]. Without proper sanitation facilities, waste from infected individuals can contaminate a community's land and water, increasing the risk of infection for other individuals [3]. Studies conducted in an urban slum of Nairobi, Kenya, and rural Odisha, India, found that fecal contamination from young children was pervasive in the domestic environment both inside and outside the household, with young children's feces being a more common source of human fecal contamination in households than older children/adults feces $[7,8]$.

The unsafe disposal of children's feces may be an important contaminant in household environments, posing a high risk of exposure to infants. Poor sanitation can result in substantial health impacts in children, including a higher prevalence of diarrhea, intestinal worms, enteropathy, malnutrition, stunting, and death. A study reported from Indonesia showed that the odds of diarrhea are significantly greater in households practicing unsafe disposal of child feces than those practicing safe disposal[9]. Similarly, another study finding reported that unsafe disposal of feces increased the risk of diarrhea by $23 \%$. It is also a risk factor for soil-transmitted helminthes infections, e.g., hookworm, ascariasis, and trichuriasis, which cause environmental diseases that are characterized by poor nutrient absorption in the gut $[6,10]$.

Previously conducted studies done on the association between unsafe disposal of child feces and reported diarrhea to have inconsistent outcomes and no tries have been made to systematically review this.Considering this, the present study aims to attract more attention to the necessity of safe child feces disposal practices and to generate evidence that can reassure changes in public policy that can encourage to address public health problems of diarrhea.

\section{Objective}

The objective of this systematic review and meta-analysis will be topool out the available evidence on the association between safe disposal of child feces and reported diarrhea in low-income and middle-income countries. The research question of this review are:

- What is the magnitude of the unsafe disposal of child feces and in low- and middle-income countries?

- Is there association between unsafe disposal of child feces and reported diarrhea in low- and middleincome countries?

\section{Methods And Analysis}




\section{Study design and Protocol}

The present study protocol is being reported following the reporting guidance provided in the PreferredReporting Items for Systematic Reviews and Meta-Analyses Protocols (PRISMA-P) statement [11] (Additional file 1)and the Preferred Reporting Items for Systematic Reviews and Meta-Analyses (PRISMA) guideline will be followed during the systematic review [12].The four phases that were drawn from the PRISMA flow chart (Figure 1) will be documented in the results to show the study selection processfrom initially identified records to finally included studies[13].The present protocol has been registered within the PROSPERO database (registration ID: CRD42020189034). Any amendments made to this protocol will be outlined and reported in the final manuscript.

\section{Criteria used for selecting studies}

Inclusion criteria for selected studies will be articles conducted in low-income and middle-income countries and written in English, articles thatinvolve under-five years of age children regardless of sex, ethnicity, and socio-economic status and observational studies (cross-sectional studies, case-control studies, and cohort studies) that examined the association between unsafe disposals of child feces and reported diarrhea.However, we will excludethe study's findings of reviews, editorials, case series and case reports, and inconsistent outcome measures.Additionally, we will exclude studies published in languages other than English, before the year 2000.

\section{Outcome of interest}

The primary outcome of this review will be pooledmagnitude of unsafe disposal of child feces and the secondary outcome of this study will be the estimate of the association of child feces disposal practices and reported diarrhea.Child feces disposal was defined based on the WHO/ UNICEF JMP for water supply \& Sanitation definition [14] as safe disposal if households responded by saying collected and disposed of in a latrine or buried it, and as unsafe disposal,if they said put into a drain or ditch, thrown into the garbage or left in the open. Whereas diarrhea defined as three or more loose or watery stools during 24 hours or any loose stool that contained blood or mucus.

\section{Information sources and search strategy}

Electronic databasessuch as PubMed, Science Direct, Cochrane Library databases,and Ovid Medline will be searched to identify relevant literature for this review. Additionally, we will hand search Google search engine, Google Scholar, and references of other studies. The literature search will be carried out by the first author in October 2020. The search strategy will be limited to studies published in English language literature. We will apply Medical Subject Headings terms from PubMed, and combined keywords to 
identify studies in the databases.We will use keywords terms and combinations with MESH terms as described below. A full search strategy for PubMed is detailed in Table 1.

Table 1. Search strategy for the PubMed database

\begin{tabular}{|c|c|}
\hline $\begin{array}{l}\mathrm{S} \\
/ \mathrm{No}\end{array}$ & Search set PubMed databases \\
\hline 1. & $\begin{array}{l}\text { "Feces"[Mesh] OR "feces"[tw] OR "faeces"[tw] OR"faecal"[tw] OR "fecal"[tw] OR "excreta"[tw] OR } \\
\text { "stool"[tw] OR "stools"[tw]] }\end{array}$ \\
\hline 2. & $\begin{array}{l}\text { dispos*[tw] OR "excrement"[tw] OR "defecation"[tw] OR manag*[tw] OR remov*[tw] OR "Waste } \\
\text { Management"[Mesh] }\end{array}$ \\
\hline 3. & $\begin{array}{l}\text { "babies"[tw] OR "baby"[tw] OR "boy"[tw] OR "boys"[tw] OR "Child, Preschool"[Mesh] OR "Child" } \\
\text { [Mesh] OR child*[tw] OR "girl"[tw] OR "girls"[tw] OR infan*"tw] OR "Infant, Newborn"[Mesh] OR } \\
\text { "Infant"[Mesh] OR "kid"[tw] OR "kids"[tw] OR "less than 5"[tw] OR "less than five"[tw] OR } \\
\text { neonat*[tw] OR new-born*[tw] OR newborn*"tw] OR "pediatric"[tw] OR pre school*[tw] OR } \\
\text { preschool*[tw] OR toddler*[tw] OR "under 5"[tw] OR "under five"[tw] OR "under fives"[tw] }\end{array}$ \\
\hline 4. & “diarrhea”[MeSH] OR “diarrhea”[tw] OR “diarrheas”[tw] OR “diarrhea”[tw] OR “diarrhoea” [tw] \\
\hline 5. & $\begin{array}{l}\text { (Africa* [tw] OR Asia*[tw] OR Caribbean* [tw] OR West Indi*[tw] OR South America*[tw] OR } \\
\text { Latin America*[tw] OR Central America*[tw]) AND("poverty"[MeSH] OR "low income } \\
\text { population"[tw]"Developing" [tw] OR less* developed[tw] OR"under developed"[tw] } \\
\text { OR"underdeveloped"[tw] OR" "middle income"[tw] OR low*income[tw] OR "underserved"[tw] } \\
\text { OR"under served"[tw] OR"deprived"[tw] OR poor*[tw])AND (countr* [tw] OR nation[tw] OR } \\
\text { population[tw] OR world[tw]) }\end{array}$ \\
\hline 6. & 1 AND 2 AND 3 AND 4 AND 5 \\
\hline
\end{tabular}

Mesh=Medical subject headings, tw=text word

\section{Study selection process}

The searched results will be export to Mendeley Desktop reference management software version 1.19.5(Mendeley Ltd., Elsevier, Netherlands) and then duplicate excluded. The screening of studies will be conducted bytwo independent review authors. The articles found by searches in databases will be evaluated for inclusion at three levels, i.e., by title, then by abstract, and finally by full-text. The full-text of selected studies will be retrieved and assessed in detail against the inclusion criteria. Discrepancies will be discussed between reviewers and refine inclusion criteria. For the screening of articles at the full-text level, rejection of an article will be decided by the review team upon the suggestion of the first reader. The details regarding the final decision of the inclusion of articles will be clarified and archived in a database. In case of uncertainty in the decision to include or exclude an article, the reviewer will include this article for the next level of screening. The documents without abstracts will be screened at the full-text level. A list of articles excluded at the full-text level will be provided in the systematic review, accompanied by reasons for exclusion. 


\section{Data extraction}

The adapted JBI data extraction formwill be used to extract the characteristics of the studies and status of child feces disposal practices and the odds ratios that showed the association of unsafe disposal of child feces and reported diarrhea. A standardized excel sheet will be created and information from the standardized review forms will be transferred to be readily available for the systematic review. For each study, authors' nameand year of publication, country of origin and set of included studies, type of study included, sample size, description of child feces disposal practices, and results of included studies will be extracted by two reviewers. Any disagreements that arise between the reviewers will be resolved through discussion or with a third reviewer.

\section{Evaluation of study quality}

The methodological quality of all studies that meet the selection criteria will be evaluated independently by two authors using the Joanna Briggs Institute (JBI) Critical Appraisal tools[15]. Each study will be assessed individually and independently by the two reviewers, both at the outcome and study level to generate an overall risk of bias score. The reviewers will assign each study as critical, serious, moderate, or low risk of bias via the judgment of the gathered information. If there is limited information, then the risk of bias will be categorized as "no information" or we will contact the corresponding authors for complete information and clarity of primary studies. The rating for each bias criterion of the two authors will then compare. Disagreements between the two authors on individual bias criteria will be identified and discussed in an attempt to reach a consensus.

\section{Data synthesis and statistical analysis}

The individual studies will be concisely described using a summary table. The summary table particularly describes the characteristics of the included studies and the main findings.Based on the summary table, we will conduct a narrative synthesis first to describe the characteristics of the included studies and the main findings. Then, meta-analysis will be conducted using a random effector fixed effect when at least three studies are comparable in design and protocol using Stata version 16 (College Station, TX 77845, USA). The overall magnitude of child feces disposal practices and its $95 \% \mathrm{Cl}$ will be calculated from standard error (SE) and magnitude of child feces disposal practices of each included study. For studies that did not present a $S E$, it will be calculated using the formula; $S E=\sqrt{ }(p \times(1-p) / n)$. The calculated SE and magnitude of each study will then be entered into Stata software to calculate the overall prevalence and its $95 \% \mathrm{Cl}$.Similarly, the association between child feces disposal practices and reported diarrhea will be summarized using statistical estimates of effect size, odds ratio (OR),and $95 \% \mathrm{Cl}$ of the study factors.We will perform subgroup analyses according to the possible characteristics of the studies andthe quality of the study included. 
Heterogeneity will be assessed statistically using a chi-square test (Q-test) for statistics and inverse variance index $\left(\mathrm{I}^{2}\right)[16] . \mathrm{I}^{2}$ values will be classified as follows: no relevant heterogeneity $(0-25 \%)$, moderate heterogeneity (25-50\%), and significant heterogeneity. The data will be considered

homogeneous if $\mathrm{I}^{2} \leq 50 \%$. Fixed effects models will be used to produce summary ORs and $95 \%$ Cls where heterogeneity did not exist. If statistical heterogeneity did exist, then random-effects models will be applied.Forest plots will be generated to present the pooled estimates with $95 \% \mathrm{Cl}$. A funnel plot and Egger's test of publication bias will be used to assess publication bias. Symmetrical funnel plot as well as insignificant Egger's test will be taken as evidence for no serious publication bias. Trim and fill techniques will be considered for substantial publication bias. A sensitivity analysis will be repeated after excluding one study to observe the impact of the individual study on the pooled estimate.

\section{Assessment of the quality of the evidence}

The quality of evidence of the outcomes will be assessed with the Grading of Recommendations, Assessment, Development, and Evaluation (GRADE) approach using GRADEPro GDT version 3.6.1 /2019 (McMaster University, ON, Canada) $[17,18]$. According to GRADE, evidence quality assessment is performed for each outcome, and the combined available evidence is considered. The GRADE approach will classify the quality of the evidence into four levels: high, moderate, low, and very low based on the comprehensive assessment of inconsistency, indirect evidence (not generalizable), inaccuracy, and publication bias. These levels represent confidence in the estimation of the treatment effects presented. The level of evidence and strength of recommendation will be determined by discussion involving all authors.

\section{Discussion}

This review and meta-analysis will systematically explore and integrate the evidence available on themagnitude of unsafe child feces disposal practices and its association with reported diarrhea. In this review, information about themagnitude and association between child feces disposal practices and reported diarrhea will be gathered and summarized. The review will be reported according to the PRISMA guidelines [13] and will be made publicly available in a repository and published in a peer-reviewed journal. The findings from this study will provide directions for future research and public health professionals with an understanding of the importance of safe child feces disposal practices to prevent childhood diarrhea in the community. The limitation of this review is that it will include only quantitative observational studies published inthe English language.

\section{Declarations}

\section{Ethics approval and consent to participate}


There is no need for an ethical assessment because we only search for and evaluate the existing literature.

Consent for publication: Not required.

Availability of data and material: All the data relevant to the study are included in the article or uploaded as supplementary information

Competing interests: None declared.

Funding: This research received no specific grant from any funding agency in the public, commercial, or not-for-profit sectors.

Authors' contributions: NES contributed to developing and drafted the protocol. NES, BKO, and DBG contributed to the supervised the development of the protocol and revise the protocol. All the authors have approved the current protocol version.

Acknowledgments: Not required.

\section{References}

1. Prüss-Ustün A, Bartram J, Clasen T, Colford JM, Cumming O, Curtis V, et al. Burden of disease from inadequate water, sanitation, and hygiene in low- and middle-income settings: A retrospective analysis of data from 145 countries. Trop Med Int Heal. 2014;19:894-905.

2. CDC - Centers for Disease Control and Prevention. Global Water, Sanitation, and Hygiene Home Healthy Water. 2017. http://www.cdc.gov/healthywater/global/. Accessed 23 Feb 2020.

3. World Health Organization. Sanitation. 2019. https://www.who.int/news-room/factsheets/detail/sanitation. Accessed 23 Feb 2020.

4. Chambers R. Going to Scale with Community-Led Total Sanitation: Reflections on Experience, Issues, and Ways Forward. IDS Pract Pap. 2009;2009:01-50. DOI:10.1111/j.2040-0225.2009.00001_2.x.

5. Prüss-Üstün, Annette; Bos, Robert; Gore F. WHO | Safer water, better health. WHO. 2014. http://www.who.int/quantifying_ehimpacts/publications/saferwater/en/. Accessed 19 Aug 2017.

6. Central Statistical Agency (CSA) [Ethiopia] and ICF. Ethiopia Demographic and Health Survey 2016: Key Indicators Report. Addis Ababa, Ethiopia, and Rockville, Maryland, USA; 2016.

7. Bauza V, Madadi V, Ocharo RM, Nguyen TH, Guest JS. Microbial source tracking using $16 \mathrm{~S}$ rRNA amplicon sequencing identifies evidence of widespread contamination from young children 's feces in an urban slum of Nairobi, Kenya. Environ Sci Technol. 2019;53:8271-8281.

8. Bauza V, Majorin F, Routray P, Sclar GD, Caruso BA, Clasen T. Child feces management practices and fecal contamination: A cross-sectional study in rural Odisha, India. Sci Total Environ.

2020;709:136169. DOl:10.1016/j.scitotenv.2019.136169. 
9. Cronin AA, Sebayang SK, Torlesse H, Nandy R. Association of Safe Disposal of Child Feces and Reported Diarrhea in Indonesia: Need for Stronger Focus on a Neglected Risk. Int J Environ Res Public Health. 2016;13:1-13.

10. Humphrey JH. Child undernutrition, tropical enteropathy, toilets, and handwashing. Lancet (London, England). 2009;374:1032-5. DOI:10.1016/S0140-6736(09)60950-8.

11. Moher D, Shamseer L, Clarke M, Ghersi D, Liberati A, Petticrew $M$, et al. Preferred reporting items for systematic review and meta-analysis protocols (PRISMA-P) 2015 statement. Syst Rev. 2015;4:1-9.

12. Moher D, Liberati A, Tetzlaff J, Altman DG, Altman D, Antes G, et al. Preferred reporting items for systematic reviews and meta-analyses: The PRISMA statement. Ann Intern Med. 2009;151:264-9.

13. Moher D, Liberati A, Tetzlaff J, Altman DG, Altman D, Antes G, et al. Preferred reporting items for systematic reviews and meta-analyses: The PRISMA statement. PLoS Med. 2009;7:889-96.

14. WHO/UNICEF. Core questions on drinking water and sanitation for household surveys. 2006.

15. Moola S, Munn Z, Tufanaru C, Aromataris E, Sears K, Sfetcu R, Currie M, Qureshi R, Mattis P, Lisy K MP-F. Chapter 7: Systematic reviews of etiology and risk - Joanna Briggs Institute Reviewers' Manual. Joanna Briggs Institute Reviewer's Manual. 2017.

16. Higgins JPT, Thompson SG. Quantifying heterogeneity in a meta-analysis. Stat Med. 2002;21:153958.

17. Guyatt GH, Oxman AD, Kunz R, Falck-Ytter Y, Vist GE L, A et al. Going from evidence to recommendations. BMJ. 2008;336:1049-51.

18. Fearns N, Kelly J, Callaghan M, Graham K, Loudon K, Harbour R, et al. GRADE guidelines: 1. IntroductiondGRADE evidence profiles and summary of findings tables. J Clin Epidemiol. 2011;64:384-94.

\section{Figures}




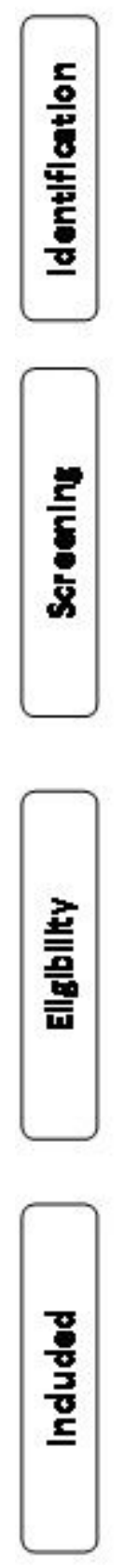

Records identified through datahase searding (n) PubMed, Science Direct, Cochrane lorary datahases, and Ovid Medine Google search engine, Google Scholar, and references of other sturies

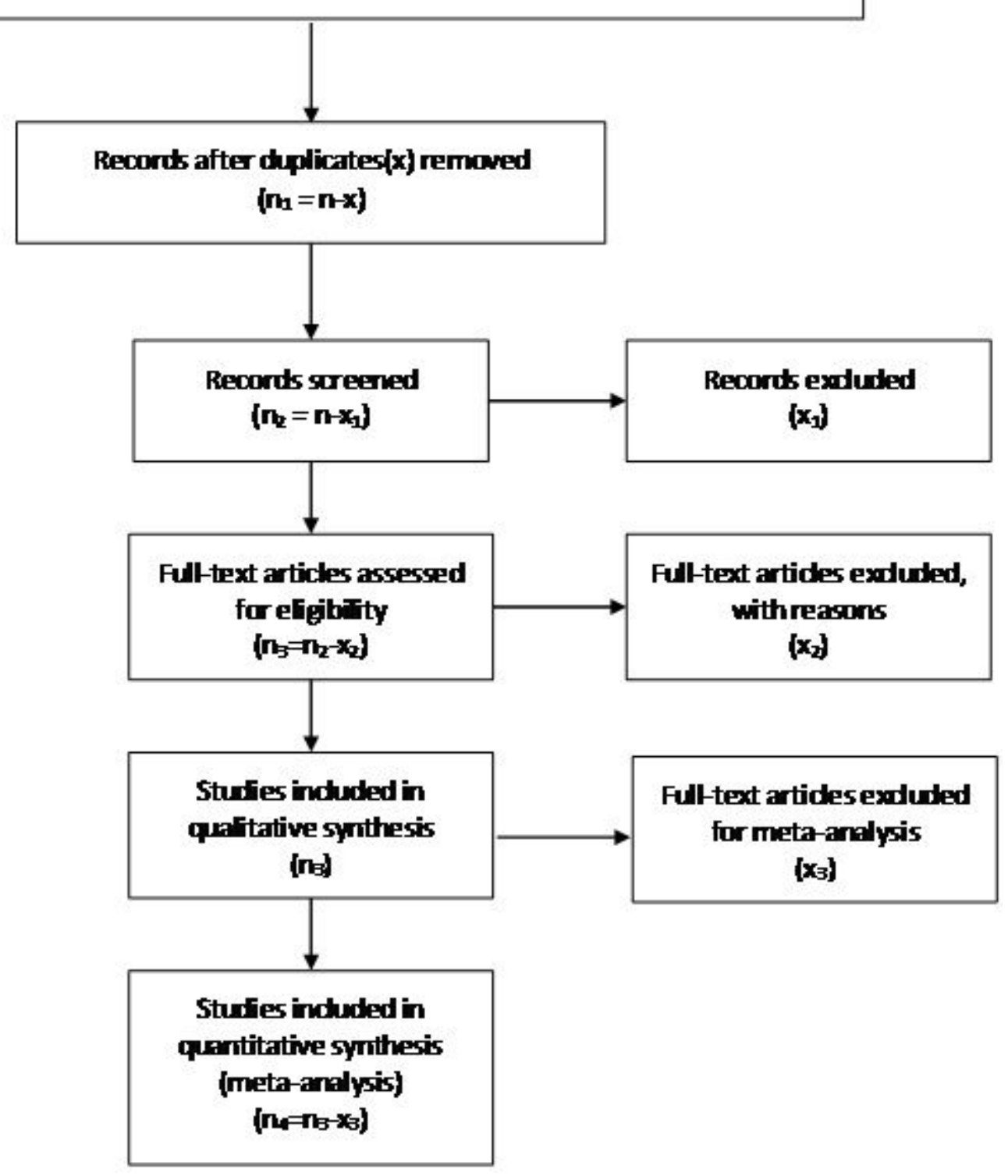

Figure 1

PRISMA flow diagram for identifying, screening, and determining the eligibility of and whether to include studies.

\section{Supplementary Files}

This is a list of supplementary files associated with this preprint. Click to download.

- Additionalfile1.docx 